\title{
Hydrogeothermal Characterization and Modelling of a Standing Column Well Experimental Installation
}

\author{
Gabrielle Beaudry
}

\author{
Philippe Pasquier
}

\author{
Denis Marcotte
}

\begin{abstract}
Standing column wells (SCW) are efficient ground heat exchangers that offer promising potential for integration in dense urban areas. Recent years have witnessed a growing interest in SCWs, resulting in the development of various simulation models incorporating heat transfer, groundwater flow and geochemical reactions within the well and the surrounding ground. However, these models commonly use a configuration that involves pumping at the bottom of the well and reinjection from its top, which can lead to installation and maintenance difficulties in deep wells. Furthermore, very few SCW models have been validated against reliable field data. This paper presents an original finite element model coupling advection-diffusion of heat and groundwater flow within a top pumped SCW and its surrounding ground as well as the experimental setup used for its validation. Within the scope of this study, experimental data obtained after an extensive field characterization campaign and a thermal response test performed with a large-scale geothermal laboratory were used directly as inputs in the numerical model. Experimental validation shows that without any calibration procedure, the model reproduces the experimental inlet and outlet groundwater temperatures with a mean absolute error of $0.14{ }^{\circ} \mathrm{C}$. It is also shown that the placement of the pump at the top of the well offers a more practical design that has minor impact on the thermal performance of the system.
\end{abstract}

\section{INTRODUCTION}

In Canada, about $17 \%$ of secondary energy use and $14 \%$ of greenhouse gases emissions are related to space heating and, albeit to a far lesser extent, cooling (NRCan 2018). As they allow for significant energy savings, groundsource heat pump systems offer great potential to alleviate the carbon footprint associated with these activities. Among those systems, standing column wells (SCW) achieve competitive heat exchange rates by recirculating groundwater in a single uncased vertical borehole, thereby minimizing the thermal resistance as well as the size and cost of the system. SCWs performance can be enhanced by discharging or bleeding part of the pumped water, an action that promotes groundwater flow and advective heat transfer around the well. This operation has been described as a key feature for maintaining the groundwater temperature within the heat pumps operational range in the peak heating conditions that frequently occur in cold climates (Spitler, et al. 2002; Deng, et al. 2005; Orio, et al. 2005; Nguyen, et al. 2015; Pasquier, et al. 2016).

Recent years have witnessed a growing interest in SCWs, resulting in the development of various simulation models incorporating heat transfer, groundwater flow (Rees, et al. 2004; Deng, et al. 2005; Abu-Nada, et al. 2008; Croteau 2011; Lee 2011; Ng, et al. 2011; Nguyen, et al. 2012, 2015) and geochemical reactions (Eppner, et al. 2015, 2017a, 2017b) within the SCW and the surrounding ground. However, these models commonly use a configuration that involves pumping at the bottom of the well where higher ground temperatures are found, and recirculation from

Gabrielle Beaudry (gabrielle.beaudry@polymtl.ca) is a Ph.D. candidate at Polytechnique Montréal, Canada.

Philippe Pasquier (philippe.pasquier@polymtl.ca) is professor of geological engineering at Polytechnique Montréal, Canada.

Denis Marcotte (denis.marcotte@polymtl.ca) is professor of geological engineering at Polytechnique Montréal, Canada. 
its top. This strategy aims at increasing the heating energy conveyed by the groundwater but can also lead to installation and maintenance difficulties in deep wells (Orio, et al. 1999). Furthermore, as some experimental projects involving SCWs exist (Minea 2013; Lee, et al. 2016; Chang, et al. 2017), only a few authors used long-term field data incorporating bleed for the validation of a predictive simulation model (Deng, et al. 2005; Ng, et al. 2011). This paper aims to present an original finite element model coupling transient advection-diffusion of heat and groundwater flow within a top pumped SCW and its surrounding ground. The experimental setup and methodology used for its validation, as well as preliminary results, will also be presented.

\section{A STANDING COLUMN WELL EXPERIMENTAL INSTALLATION}

As part of an ongoing research program on SCWs in cold climates, a mobile geothermal laboratory (MGL) was assembled in a 12.2 meter marine container located in the city of Varennes at $10 \mathrm{~km}$ east of Montreal (Canada) in the spring of 2016. The MGL was designed to mimic the heating and cooling operation of a small commercial building connected to a borehole heat exchanger (closed or open-loop, vertical or horizontal) so as to obtain detailed information about the thermo-hydro-geochemical processes occurring during the operation of open and closed-loop ground heat exchangers. Accordingly, the MGL was equipped with a heating, ventilation and air-conditioning system allowing heat extraction or injection through four $14 \mathrm{~kW}$ water-to-air heat pumps connected in a parallel arrangement with a propylene glycol loop. A plate heat exchanger connects the propylene glycol loop to the groundwater loop to prevent fouling in the heat pumps. In addition, the MGL has a $24 \mathrm{~kW}$ nominal water heater and a water treatment unit designed to condition groundwater chemistry. A comprehensive control and data acquisition system monitoring the electrical power, flow rate, fluid and air temperatures every minute is also put into place in the MGL.

Next to the MGL, a $300 \mathrm{~m}$ deep SCW (Ø165 mm) and a $150 \mathrm{~m}$ deep injection well (IW) (Ø165 mm) allowing to return the bleed water to the aquifer were drilled at about $10 \mathrm{~m}$ from each other in June 2016. Later in the fall, the wells were equipped with high-density polyethylene pumping and reinjection pipes (SDR 11, Ø50.8 mm) and with distributed fibre optic temperature sensors. The installation delay caused the SCW bottom walls to collapse, resulting in an effective depth of $215 \mathrm{~m}$. For practical and financial considerations, the submersible pump was located near the surface in a pumping chamber $(\varnothing 241 \mathrm{~mm})$ at a depth of about $18 \mathrm{~m}$. Figure 1 illustrates the MGL setup.
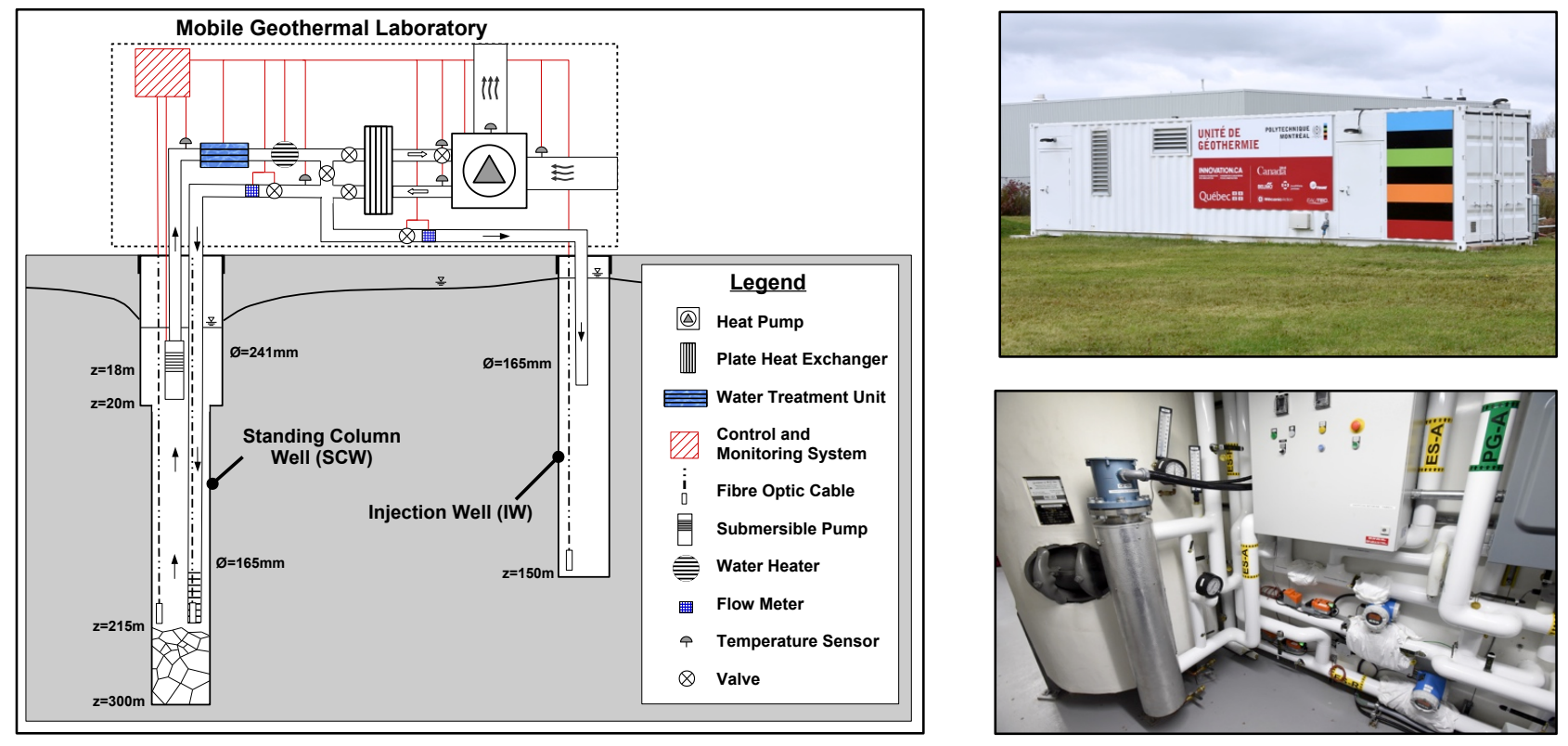

Figure 1 Schematic illustration of the MGL (not to scale) (left), MGL viewed from outside (upper right) and experimental apparatus used for the thermal response test: water heater, flow meters, valves and temperature sensors (lower right). 


\section{CHARACTERIZATION OF THE EXPERIMENTAL SITE}

A comprehensive characterization of the site was undertaken in 2017 to acquire detailed information about aquifer characteristics. Parameters needed for the development of an accurate numerical model and its validation, namely, the nature, depth and temperature of the bedrock, groundwater static hydraulic head, aquifer specific storage, hydraulic and thermal conductivity, were obtained following the tests listed below.

\section{Geology}

A first evaluation of the site hydrogeothermal conditions was made based on local geology. A stratigraphic column was developed following the macroscopic analysis of 54 composite samples coming from drill cuttings, each representing $6.1 \mathrm{~m}$ along the SCW. The bedrock was identified at a depth of $3 \mathrm{~m}$ and corresponds to a grey finegrained sedimentary rock intersected locally by beds of siltstone, beige-pink sandstone and beige limestone as well as intermediate to mafic sills and dikes. A gradual transition was observed after a depth of $215 \mathrm{~m}$ that leads to a black shaly limestone at a depth of $294 \mathrm{~m}$. Those observations are consistent with regional stratigraphic sequence (Globensky 1987) and correspond, respectively, to the Nicolet Formation and Utica Group of the Ordovician period.

\section{Temperature Profiles}

As the local geothermal heat flux and far-field thermal conditions affect groundwater temperatures and thereby SCWs performance, a temperature profile was measured prior to any heating or cooling activity. Temperature sensors with $0.25{ }^{\circ} \mathrm{C}$ accuracy and 20 seconds response time were taken down the wells with a thermal stabilization period of 60 seconds between each displacement. Shallow temperatures in the borehole $(0-30 \mathrm{~m})$ showed significant variations attributable to seasonal air temperature fluctuations. A geothermal gradient of $2.3^{\circ} \mathrm{C} / 100 \mathrm{~m}$ associated with the local geothermal heat flux was observed from $60 \mathrm{~m}$ depth $\left(8.9^{\circ} \mathrm{C}\right)$ down to $215 \mathrm{~m}\left(12.4^{\circ} \mathrm{C}\right)$.

\section{Hydrogeology}

To identify aquifer characteristics and gain better understanding of local groundwater flow, a 6-day pumping test was conducted in October 2017. Groundwater was discharged from the SCW to a nearby storm sewer at a rate of $33 \mathrm{~L} / \mathrm{min}$ to reflect a typical maximal bleed ratio of 30\% (Orio, et al. 2005; Pasquier, et al. 2016). The water level was located at an initial depth of $2 \mathrm{~m}$ and was monitored during the test using pressure sensors with $0.5 \mathrm{cmH}_{2} \mathrm{O}$ accuracy. Upon the test ending, stabilized drawdowns of about $4 \mathrm{~m}$ and $2 \mathrm{~m}$ were observed, respectively, in the pumping well (SCW) and non-operating IW. These stabilized drawdowns suggest the presence of a recharge boundary nearby that is yet unlocalized. Given the very friable nature of the bedrock along the effective borehole depth, groundwater flow was considered to be taking place in a homogeneous equivalent porous medium. On this basis, hydraulic parameters estimates were obtained from the Cooper and Jacob (1946) solution for confined aquifers after analysis of the drawdowns at the SCW and IW (see Figure 2). Slightly different hydraulic conductivity values resulted from the SCW $\left(5.7 \mathrm{e}-7 \mathrm{~m} \mathrm{~s}^{-1}\right)$ and IW $\left(7.8 \mathrm{e}-7 \mathrm{~m} \mathrm{~s}^{-1}\right)$, which may reflect heterogeneity of the fractured aquifer. Both values are typical of a moderately permeable sedimentary bedrock (Freeze and Cherry 1979). Specific storage coefficients of $6.4 \mathrm{e}-4 \mathrm{~m}^{-1}$ $(\mathrm{SCW})$ and $7.5 \mathrm{e}-7 \mathrm{~m}^{-1}(\mathrm{IW})$ were obtained. The latter avoids wellbore storage effects and indicates a confined aquifer.

\section{Geothermal}

Thermal conductivity of surrounding rocks has been identified as one of the most influential factors affecting SCWs performance alongside borehole length and bleed ratio (Deng, et al. 2005). Hence, this parameter was tested under both laboratory and field conditions. Thermal needle probe (TNP) tests were first carried out on the saturated drill cuttings samples in March 2017. The procedure was performed following ASTM D-5334-08 protocol (ASTM 2008) with a Hukseflux ${ }^{\text {TM }}$ needle thermal conductivimeter. 
A thermal response test (TRT) was also conducted in November 2017 to determine the in situ thermal conductivity accounting for conductive as well as advective heat transfer mechanisms. After recirculating groundwater in the SCW for 24 hours to measure initial ground temperature, heat was injected in the borehole for six days using a water heater having a $24 \mathrm{~kW}$ nominal capacity, followed by a three-day recovery phase. During the TRT, water was recirculated in the SCW without bleed and flow rate was maintained around $98 \mathrm{~L} / \mathrm{min}$. Entering (EWT) and leaving (LWT) groundwater temperatures were monitored at the inlet and outlet of the water heater with temperature sensors having $0.2^{\circ} \mathrm{C}$ accuracy. The heating power $Q$ was maintained around $23.4 \mathrm{~kW}$ assuming that $Q=\Delta T \dot{V}\left(\rho C_{p}\right)_{w}$, where $\Delta T$ is the difference between the EWT and LWT, $\dot{V}$ is the pumping rate and $\rho C_{p}$ is the water volumetric heat capacity. This value implies a correction that accounts for the heat loss in the horizontal pipes between the well and the MGL.

Both tests were interpreted using Kelvin's infinite line source theory (Mogensen 1983), where the temperature rise only depends on the heating power and thermal conductivity $k$. This approximation is valid after a short transient period represented by $t \geq 5 r_{b}{ }^{2} / \alpha$ :

$$
k_{e q}=\left(\frac{q}{4 \pi \Delta T}\right) \ln \left(\frac{t_{2}}{t_{1}}\right) \text { with } k_{e q, \text { betero }}=\prod_{i=1}^{N} k_{i}^{\phi_{i}} \text { and } k_{e q, \text { fractured }}=\sum_{i=1}^{N} \phi_{i} k_{i}
$$

where $\alpha=k / \rho C_{p}$ is the thermal diffusivity, $r_{b}$ is the borehole radius, $q$ is the specific heat load and $\Delta T$ is the temperature change between time $t_{1}$ and $t_{2}$. $k_{e q}$ represents the equivalent thermal conductivity of a composite medium as a function of the volumetric fraction $\Phi_{i}$ and the thermal conductivity $k_{i}$ of $N$ components. Regarding the TNP tests, the saturated cutting samples were considered a heterogeneous medium composed of rock, $3 \%$ of trapped air, and a variable water volumetric fraction estimated after stove-drying the samples. As for the TRT, the in situ saturated bedrock was assimilated to a fractured medium with an effective porosity of $2 \%$, which is typical of fine-grained and poorly fissured sedimentary rock (Bear and Cheng 2010).
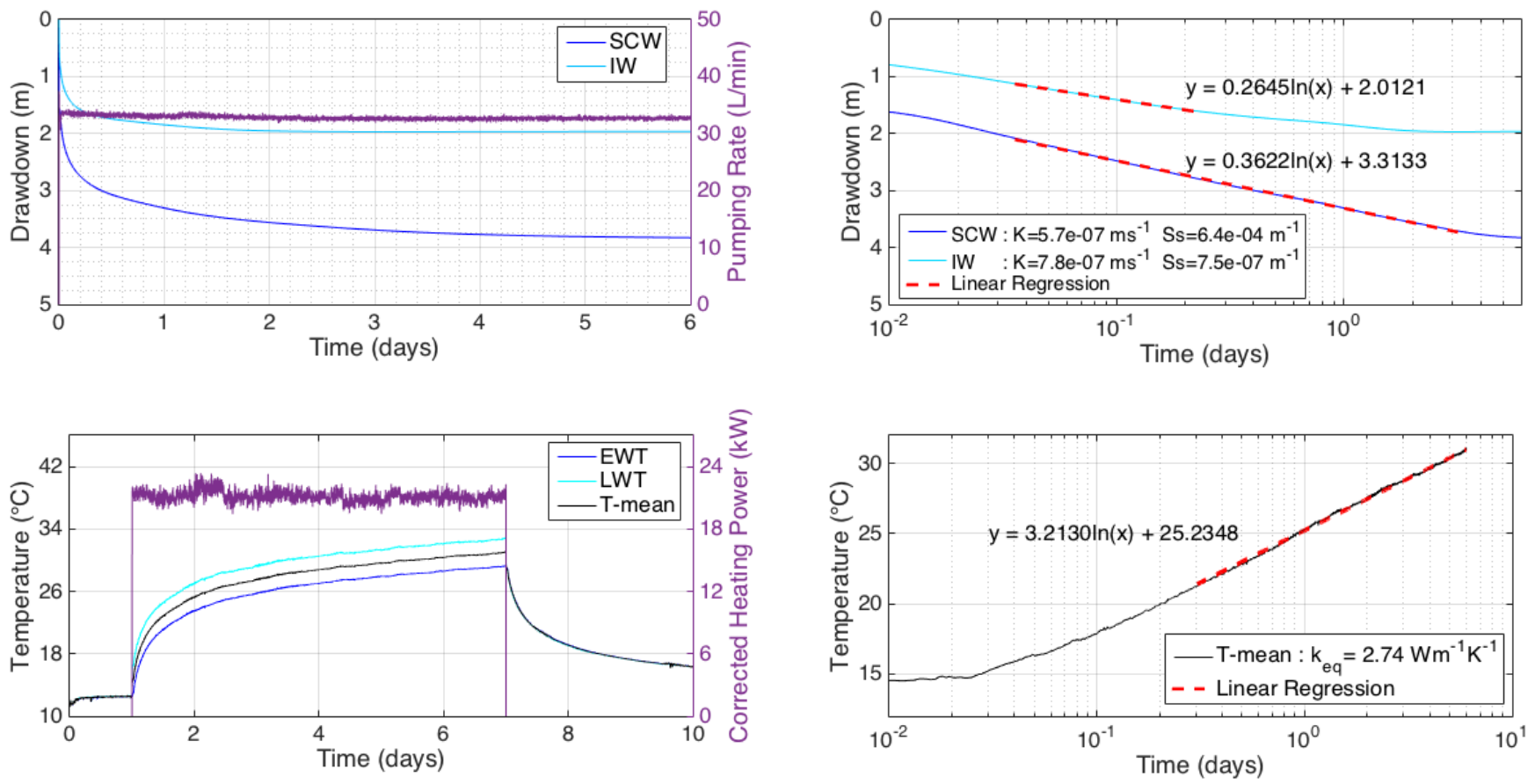

Figure 2 Pumping test data (upper left) and analysis (upper right); Thermal response test data (lower left) and analysis (lower right). 
The mean thermal conductivity of the rock fragments was estimated to be $2.49 \mathrm{Wm}^{-1} \mathrm{~K}^{-1}$ by the TNP tests performed in laboratory. The in situ value was rather evaluated at $2.74 \mathrm{Wm}^{-1} \mathrm{~K}^{-1}$ and $2.78 \mathrm{Wm}^{-1} \mathrm{~K}^{-1}$, respectively, for the equivalent fractured medium and the rock matrix (see Figure 2). A derivative-based analysis confirmed these values (Pasquier 2018). The difference between the laboratory and in situ values is minor and can be attributed to the (1) different borehole length tested by the TNP tests $(305 \mathrm{~m})$ and the TRT $(215 \mathrm{~m}),(2)$ advective heat transfer promoted by the pumping action, (3) scale effects and (4) modified water content of the laboratory samples. Note that no temperature fluctuation was observed in the IW during the test.

\section{NUMERICAL MODELLING STRATEGY}

Modelling the complex hydrogeothermal processes occurring in SCWs operation can be achieved by coupling a groundwater flow model and a heat transfer model in the Comsol Multiphysics environment (Nguyen, et al. 2012; Eppner, et al. 2015, 2017a, 2017b). In the present work, the SCW geometry illustrated in Figure 1 was represented by a simplified 2D axisymmetric configuration in order to minimize computation time. Accordingly, the riser pipe was adapted to a concentric annular space of equivalent cross-sectional area. The model dimensions are listed in Table 2.

Table 2. Geometry of the Numerical Model

\begin{tabular}{cccccc}
\hline Parameter & Aquifer & Upper SCW & Lower SCW & Riser Pipe & Reinjection Pipe \\
\hline \hline Depth & $215 \mathrm{~m}$ & $20 \mathrm{~m}$ & $215 \mathrm{~m}$ & $18 \mathrm{~m}$ & $215 \mathrm{~m}$ \\
Radius & $10 \mathrm{~m}$ & $121 \mathrm{~mm}$ & $83 \mathrm{~mm}$ & $44.58 \mathrm{~mm}$ (outer) & $30.16 \mathrm{~mm}$ (outer) \\
Wall thickness & - & - & - & $5.82 \mathrm{~mm}$ & $5.82 \mathrm{~mm}$ \\
\hline
\end{tabular}

The geometrical elements were defined with three distinct materials representing the aquifer, the HDPE pipes and groundwater. The experimental hydraulic and thermal properties provided by the field investigation were directly attributed to the aquifer domain. More specifically, the hydraulic properties derived from the SCW data and the in situ thermal conductivity of the rock matrix obtained from the TRT analysis were selected. Typical values for porosity and volumetric heat capacity of most rocks (Banks 2008) were also assigned to the aquifer domain. The pipes and groundwater thermal parameters were obtained respectively from the manufacturer's specifications and the reported standard values at $20^{\circ} \mathrm{C}$ (see Table 3). The hydraulic paramaters were attributed to represent, respectively, a perfectly impervious solid and a perfectly pervious liquid with very low storage.

Table 3. Hydraulic and Thermal Properties of Model Materials

\begin{tabular}{cccccc}
\hline Parameter & Symbol & Unit & Aquifer & Pipe & Water \\
\hline \hline Volumetric Heat Capacity & $\rho C_{p}$ & $\mathrm{~kJ} \mathrm{~m}^{-3} \mathrm{~K}^{-1}$ & 2070 & 2174 & 4176 \\
Porosity & $n$ & - & 0.02 & $1 \mathrm{e}-5$ & 1 \\
Thermal Conductivity & $k$ & $\mathrm{~W} \mathrm{~m}^{-1} \mathrm{~K}^{-1}$ & $2.78^{*}$ & 0.42 & 0.59 \\
Hydraulic Conductivity & $K$ & $\mathrm{~m} \mathrm{~s}^{-1}$ & $5.7 \mathrm{e}-7^{*}$ & $1 \mathrm{e}-9$ & 1000 \\
Specific storage & $S_{s}$ & $\mathrm{~m}^{-1}$ & $6.4 \mathrm{e}-4 *$ & $4 \mathrm{e}-10$ & $4 \mathrm{e}-10$ \\
\hline
\end{tabular}

\section{Governing Equations}

*Values provided by the detailed field investigation

Groundwater motion in the model is assimilated to a saturated flow within an equivalent porous medium. Hence, the governing equations are the continuity equation and Darcy's law as expressed by Equation 2, where $\rho$ is the fluid density, $g$ is the gravitational acceleration, $p$ is the pressure, $u$ is the Darcy velocity, $K$ is the hydraulic conductivity, $z$ is the elevation and $S_{s}$ is the specific storage :

$$
\rho S_{s} \frac{\partial p}{\partial t}=-\nabla \cdot(\rho u) \text { and } u=\frac{-K}{\rho g}\left(\nabla p+\rho g \nabla_{z}\right)
$$


Thermal effects in the developed model consider conductive and advective heat transfer mechanisms. The latter accounts for the motion of the water within the well and the surrounding aquifer, and depends on the Darcy velocity computed by Equation 2. The state equation solved for heat transfer is given by Equation 3, where $T$ is the groundwater temperature and $k_{e q}$ and $\left(\rho C_{p}\right)_{e q}$ are respectively the thermal conductivity and volumetric heat capacity of the equivalent porous medium :

$$
\left(\rho C_{p}\right)_{e q} \frac{\partial T}{\partial t}+\left(\rho C_{p}\right)_{w} u \cdot \nabla T=\nabla \cdot\left(k_{e q} \nabla T\right)
$$

\section{Initial and Boundary Conditions}

Boundary conditions used in the model are illustrated in Figure 3. The confined aquifer roof was located $3 \mathrm{~m}$ below the surface at the soil-bedrock interface. A constant hydraulic head of $213 \mathrm{~m}$ relative to the base of the borehole was set as an initial head for the domain and along the lateral boundary. As the ground below the SCW was not explicitly represented in the model for efficiency purposes, both inflow and outflow boundaries located at the top and the bottom of the well were attributed a normal velocity $v=\dot{V}(1-\beta) / A$ derived from the pumping rate $\dot{V}$, the corresponding cross-sectional area $A$ and a bleed ratio $\beta$. The latter was fixed at zero for the riser pipe top boundary.

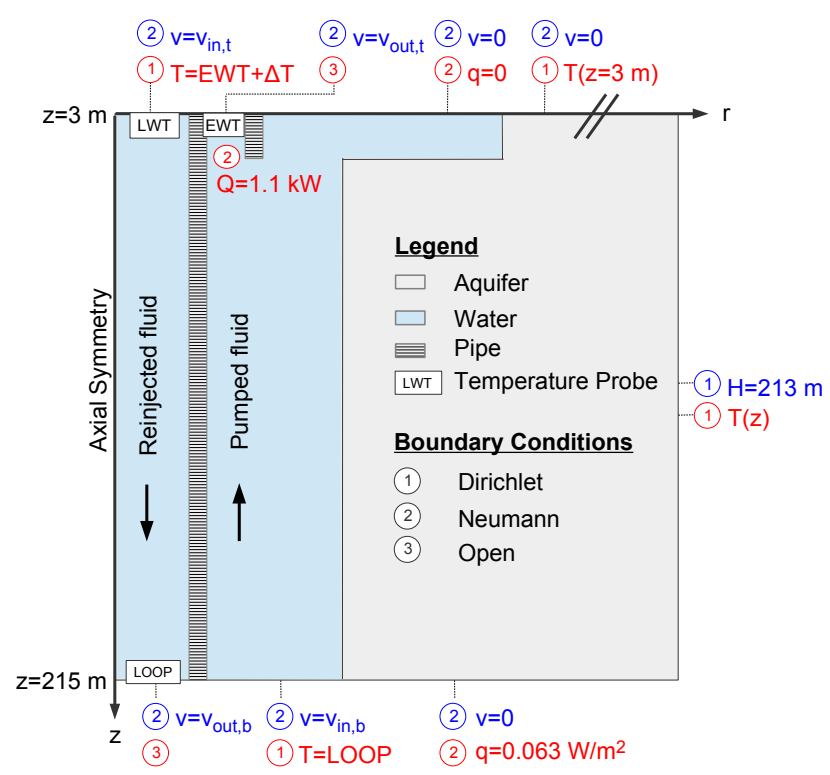

Figure 3 Boundary conditions of the hydraulic (blue) and heat transfer (red) models.

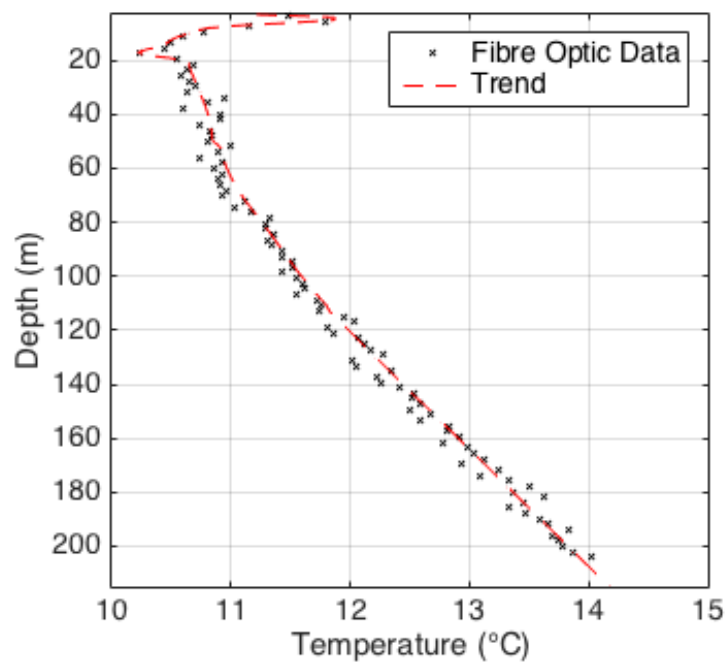

Figure 4 Initial solution and lateral boundary condition $\mathrm{T}(\mathrm{z})$ used in the simulation.

As for the thermal conditions, a natural geothermal heat flux $\left(0.063 \mathrm{~W} \mathrm{~m}^{-2}\right)$ was derived from the equivalent thermal conductivity of the aquifer and the geothermal gradient, and then set as the specific heat load $q$ at the base of the model. The initial solution on the whole simulation domain and temperatures along the outer radial boundary and the aquifer roof were intended to correspond to vertical temperature profiles measured in the SCW (T(z)). The latter, as well as the thermal load $Q$ imposed by the MGL apparatus (the heat pumps or the water heater) can be implemented in the finite element model via interpolation functions. The temperature variation $\Delta T$ induced by the thermal load is computed by the model at each time step and considered as the difference between the outlet and intlet fluid temperatures: 


$$
L W T=E W T+\Delta T \text { with } \Delta T=Q /\left(\dot{V}\left(\rho C_{p}\right)_{w}\right)
$$

where EWT is integrated along the riser pipe upper boundary. The mean temperature along the well lower outflow boundary is also integrated and then imposed on the adjacent inflow boundary. Ultimately, a heat source equivalent to the power consumption of the submersible pump was set in the annular region at a depth of $18 \mathrm{~m}$.

\section{EXPERIMENTAL VALIDATION OF THE NUMERICAL MODEL}

A simple simulation reproducing the previously described TRT was conducted to validate the numerical model. It is important to note that the parameters derived from the field study were directly implemented in the model and that no calibration procedure was performed. The experimental heating loads and pumping rates were also directly implemented at intervals of one minute. A temperature profile measured in the SCW 15 minutes before the test was set as the temperature initial solution on the whole domain and imposed at the lateral boundary and aquifer roof (see Figure 4). This simulation was carried out using a maximum time step of one minute. The difference between the simulated and the experimental temperatures is analyzed and the mean absolute error (MAE) is calculated. Figure 5 shows a very good agreement between experimental and computed groundwater temperatures without any model calibration. The resulting MAE is $0.14{ }^{\circ} \mathrm{C}$.

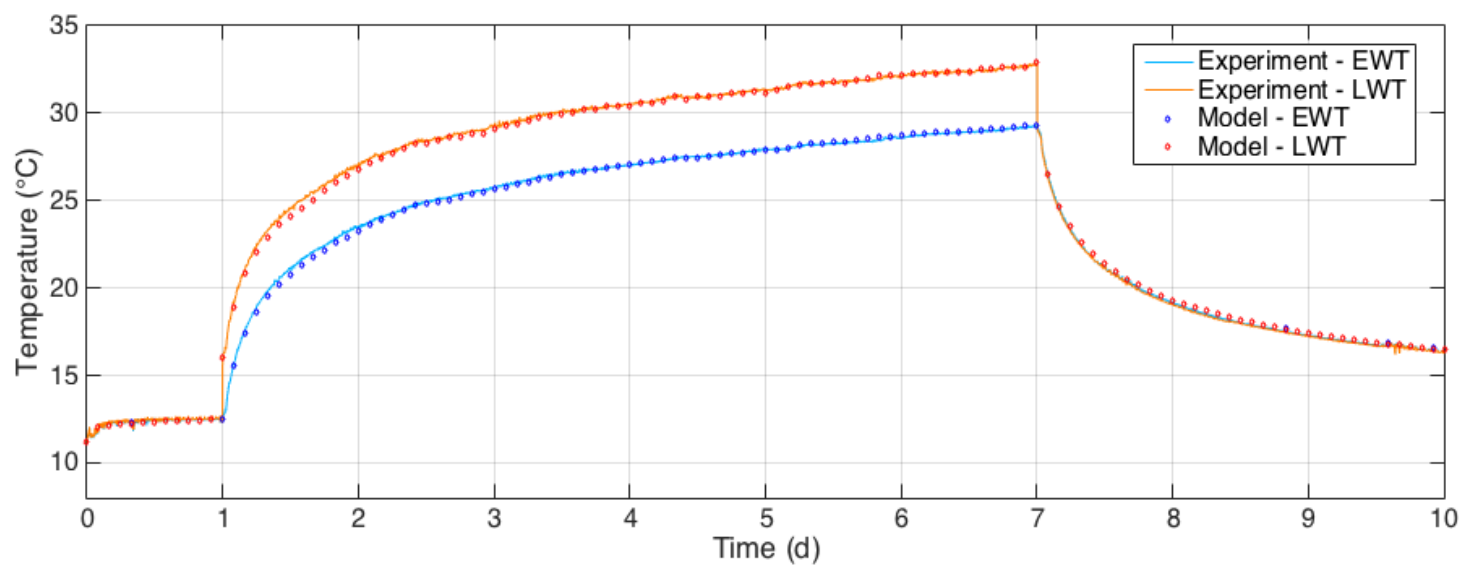

Figure 5 Comparison of EWT and LWT as computed by the numerical model and measured experimental.

\section{FURTHER RESULTS AND DISCUSSION}

The modelling strategy described in this work was developed with the intent to reproduce the hydrogeothermal behaviour of an experimental SCW installation. Hence, the real system dimensions and materials were reproduced with minimal simplifications aiming to lighten the computation burden. The ground hydrogeothermal properties obtained from an independent in situ investigation of the SCW surroundings were also directly implemented in the model. A simple simulation of the TRT showed that this strategy allows for the computation of groundwater temperatures at the inlet and outlet of the well with a MAE of $0.14{ }^{\circ} \mathrm{C}$, which is smaller than the accuracy of the temperature sensor $\left(0.2^{\circ} \mathrm{C}\right)$.

It was previously mentioned that there is a minor difference between the rock thermal conductivity $(2.78 \mathrm{~W}$ $\left.\mathrm{m}^{-1} \mathrm{~K}^{-1}\right)$ derived from the in situ TRT - which was used for the experimental validation of the model - and the laboratory TNP tests $\left(2.49 \mathrm{~W} \mathrm{~m}^{-1} \mathrm{~K}^{-1}\right)$. In order to evaluate the impact of this variation on the temperatures computed 
by the model, a comparative simulation was carried out using the second value. The resulting inlet and outlet groundwater temperatures were distinctly overestimated and a MAE of $1.04{ }^{\circ} \mathrm{C}$ was calculated between the model response and the experimental data. Calibration of the volumetric heat capacity value within a realistic range did not allow a satisfying agreement. These results suggest that a slight variation in ground properties estimates can be a significant source of error and emphasize the advantages conferred by in situ tests when evaluating the local values.

In the scope of this study, it was also proposed to evaluate the influence of the pump placement on the performance of the system. A quick modification to the model boundaries allowed for the switch of the pump and reinjection depths, therefore placing the pump inlet at the bottom of the well $(215 \mathrm{~m})$ and the reinjection at a depth of $18 \mathrm{~m}$. The TRT simulation was then repeated. Results showed minimal deviation (MAE of $0.12{ }^{\circ} \mathrm{C}$ ) from the inlet and outlet groundwater temperatures computed by the previous top-pumped simulation presented in Figure 5. These results suggest that installation and maintenance difficulties occasioned by the widespread down-pumped configuration of SCWs (Orio, et al. 2005; Pasquier, et al. 2016) could be avoided by placing the submersible pump in the annular region at the top of the well since both configurations provide, in the absence of bleed, the same EWT.

\section{CONCLUSION}

In this paper, a finite element model coupling transient advection-diffusion of heat and groundwater flow was developed to simulate the behaviour of an experimental SCW connected to a large-scale geothermal laboratory. The thermal conductivity, hydraulic conductivity and specific storage of the aquifer were identified by a comprehensive characterization campaign and were directly integrated in the model. Results of a simple simulation reproducing an in situ thermal response test showed that without any calibration procedure, the model reproduces the experimental EWT and LWT with a MAE of $0.14{ }^{\circ} \mathrm{C}$. Further simulations also suggest that the pump inlet depth has little impact on the well heat exchange efficiency in the absence of bleed. Ongoing research activities and incoming experimental data from the MGL operation in the heating and cooling mode should allow further development of the model so as to include the impact of bleed and the potential hydrogeothermal interaction with a nearby injection well.

\section{ACKNOWLEDGMENTS}

The authors acknowledge the financial support by Institut de l'Énergie Trottier (IET) and would like to thank Étienne Bélanger and Pierre Beaudry for their technical support.

\section{NOMENCLATURE}
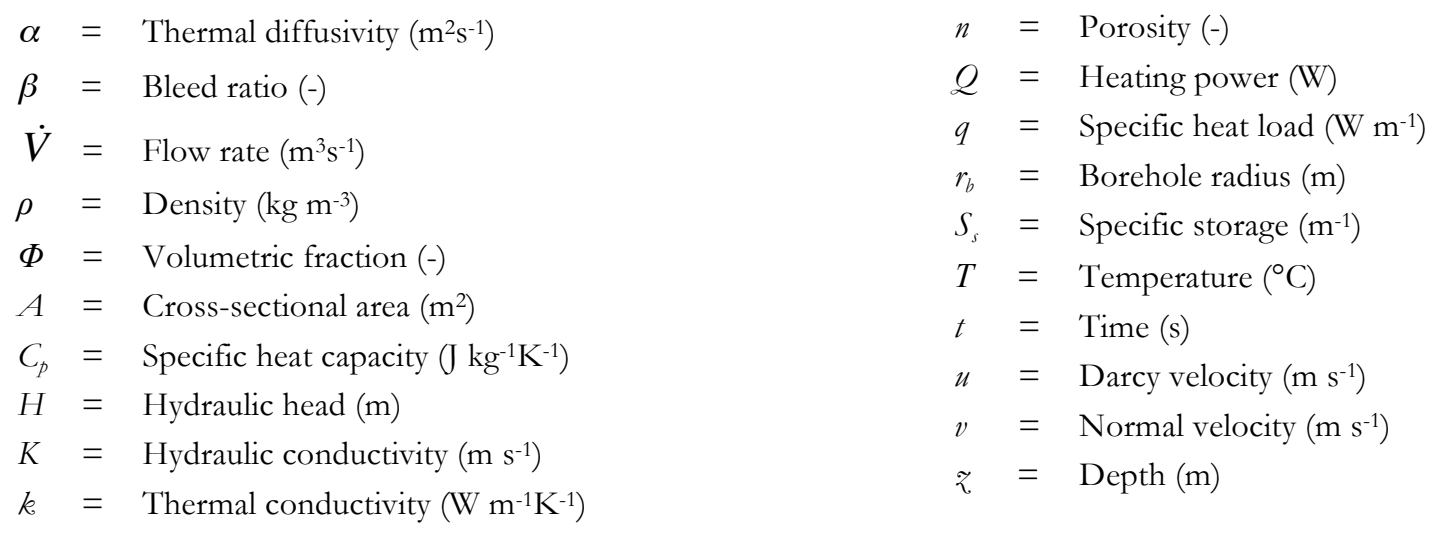

\section{Subscripts}
$b=$ bottom
$t=$ top
$e q=$ equivalent
$w=$ water 


\section{REFERENCES}

Abu-Nada, E., Akash, B., Al-Hinti, I., Al-Sarkhi, A., Nijmeh, S., Ibrahim, A., \& Shishan, A. (2008). Modeling of a geothermal standing column well. International Journal of Energy Research, 32(4), 306-317.

ASTM International. (2008). D5334-08 Standard Test Method for Determination of Thermal Conductivity of Soil and Soft Rock by Thermal Needle Probe Procedure. ASTM International.

Banks, D. (2008). An introduction to thermogeology: ground source heating and cooling. Oxford: Blackwell.

Bear, J., \& Cheng, A. H. D. (2010). Modeling groundwater flow and contaminant transport (Vol. 23). Springer Science \& Business Media.

Chang, K. S., Kim, M. J., \& Kim, Y. J. (2017). An Experimental Study on the Thermal Performance Evaluation of SCW Ground Heat Exchanger. International Journal of Air-Conditioning and Refrigeration, 25(01), 1750006.

Comsol Multiphysics. (2013). Version 4.4, COMSOL.

Cooper, H. H., \& Jacob, C. E. (1946). A generalized graphical method for evaluating formation constants and summarizing well-field history. Eos, Transactions American Geophysical Union, 27(4), 526-534.

Croteau, J. É. (2011). Évaluation des paramètres influençant les températures d'opération des puits à colonne permanente. École Polytechnique de Montréal.

Deng, Z., Rees, S. J., \& Spitler, J. D. (2005). A model for annual simulation of standing column well ground heat exchangers. HVAC\&R Research, 11(4), 637-655.

Eppner, F., Pasquier, P., Baudron, P. (2015). Development of a Thermo-Hydro-Geochemical Model for Low Temperature Geoexchange Applications. In Comsol Conference, Boston, US A.

Eppner, F., Pasquier, P., \& Baudron, P. (2017). A coupled thermo-hydro-geochemical model for standing column well subject to CO2 degassing and installed in fractured calcareous aquifers. Geomechanics for Energy and the Environment.

Eppner, F., Pasquier, P., \& Baudron, P. (2017). Investigation of Thermo-hydro-geochemical Processes in a Standing Column Well Intersected by a Fracture. In Proceedings of the IGSHPA Technical/Research Conference and Expo.

Freeze, R. A., \& Cherry, J. A. (1979). Groundwater, 604 pp. Prentice-Hall, Englewood Cliffs, NJ.

Globensky, Y. (1987). Géologie des Basses-Terres du St-Laurent (Geology of the St. Lawrence Lowlands). Ministry of Energy and Resources, General Direction for Geologic and Mineral Exploration, Québec, Report MM, 85-02.

Hukseflux Therml Sensors. (n.d.). Multi-purpose Thermal Needle System for Thermal Resistivity/Conductivity Measurment. Hukseflux Thermal Sensors.

Lee, K. S. (2011). Modeling on the performance of standing column wells during continuous operation under regional groundwater flow. International Journal of Green Energy, 8(4), 474-485.

Lee, S., Lee, C., Moon, H., Jeong, J., Lee, Y., Kim, H., Jo, S. (2016). Thermal response performance of the heat exchanger of a standing column well based on the location of the return pipe. JP Journal of Heat and Mass Transfer, 13(4), 559.

Minea, V. (2013). Experimental investigation of the reliability of residential standing column heat pump systems without bleed in cold climates. Applied Thermal Engineering, 52(1), 230-243.

Mogensen, P. (1983). Fluid to duct wall heat transfer in duct system heat storages. Document-Swedish Council for Building Research, (16), 652-657.

Natural Resources Canada. (2018). Energy Use Data Handbook. Retrieved from :

http://oee.nrcan.gc.ca/corporate/ statistics $/$ neud $/ \mathrm{dpa} /$ showTable.cfm?type $=H B \&$ sector $=$ aaa\&juris $=$ ca\&rn $=2 \&$ page $=0$

Ng, B. M., Underwood, C. P., \& Walker, S. L. (2011). Standing column wells-Modeling the potential for Applications in Geothermal Heating and Cooling. HVAC\&R Research, 17(6), 1089-1100.

Nguyen, A., Pasquier, P., \& Marcotte, D. (2012). Multiphysics modelling of standing column well and implementation of heat pumps off-loading sequence. In Comsol Conference, Boston, US A.

Nguyen, A., Pasquier, P., \& Marcotte, D. (2015). Thermal resistance and capacity model for standing column wells operating under a bleed control. Renewable Energy, 76, 743-756.

Orio, C. D., Chiasson, A., Johnson, C. N., Deng, Z., Rees, S. J., \& Spitler, J. D. (2005). A Survey of Standing Column Well Installations in North America. ASHRAE Transactions, 111(2).

Pasquier, P. (2018). Interpretation of the first hours of a thermal response test using the time derivative of the temperature. Applied Energy, 213, 56-75.

Pasquier, P., Nguyen, A., Eppner, F., Marcotte, D., \& Baudron, P. (2016). Standing column wells. In Advances in GroundSource Heat Pump Systems (pp. 269-294). Elsevier. 
Rees, S. J., Spitler, J., Deng, Z., Orio, C., \& Johnson, C. (2004). A study of geothermal heat pump and standing column well performance. ASHRAE Transactions, 110(1), 3-13.

Spitler, J. D., Rees, S. J., Deng, Z., Chiasson, A., Orio, C. D., \& Johnson, C. (2002). R\&D studies applied to standing column well design. ASHRAE Research Project. 